\title{
Multi-agent System for Recognition of Hand Postures
}

\author{
Mariusz Flasiński, Janusz Jurek, and Szymon Myśliński \\ IT Systems Department, Jagiellonian University \\ Straszewskiego 27, 31-110 Cracow, Poland \\ http://www.wzks.uj.edu.pl/ksi
}

\begin{abstract}
A multi-agent system for a recognition of hand postures of the Polish Sign Language is presented in the paper. The system is based on a syntactic pattern recognition approach, namely on parsable ETPL $(k)$ graph grammars. An occurrence of a variety of styles of performing hand postures requires an introduction of many grammar productions that differ each from other slightly. This makes a construction of a grammar within a parsable class ETPL(k) dubious. Dividing a whole grammar into sub-grammars and distributing them to agents allows one to solve the problem.
\end{abstract}

Keywords: pattern recognition, multi-agent system, ETPL(k) graph grammar.

\section{Introduction}

Multi-agent systems are used as a useful approach in a pattern recognition area [11 1321]. It concerns, especially, real-time applications. However, real-time requirements make a construction of multi-agent systems difficult 152022 . The results of our recent research has revealed that a syntactic pattern recognition paradigm together with a multi-agent system approach gives a convenient basis for solving real-time pattern recognition problems 91617. At the same time, it seems that in an aspect of an efficiency of a symbolic description processing it performs better than many other computationally inefficient standard artificial intelligence methods 19 .

The main idea of syntactic pattern recognition consists in treating a pattern as a structure of the form of string, tree or graph. A set of patterns to be recognized is treated, in turn, as a formal language (string language, tree language or graph language) that is generated with a formal grammar. A formal automaton (syntax analyzer, parser) constructed for the grammar is then used as a recognition algorithm. The ETPL $(k)$ graph grammars (Embedding Transformation-

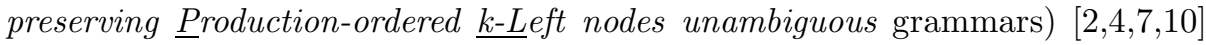
have been used as a syntactic pattern recognition model for a variety of applications such, as: a pattern recognition in the industrial robot control system [4, an analysis of a distributed environment configuration in the software allocation system [3], a solid modelling and analysis in the CAD/CAM (Computer Aided 
Design / Computer Aided Manufacturing) integration system [5], a solid representation for an optimal scaling in the CAE (Computer Aided Engineering) parallel computation system [6], an analysis of a complex experimental physics equipment in the real-time expert control system [816. Analogously like in the last mentioned area (real-time expert control system), we have applied a multiagent approach during our recent research concerning a real-time recognition of hand postures occurring in the Polish Sign Language. The results of the research are presented in the paper.

Let us notice that pattern recognition of hand postures is widely investigated all over the world [121418. The methods of hand posture recognition can be applied in the field of gesture recognition [118]. A distributed recognition approach is also widely used for recognition tasks to boost system performance and/or recognition rate 12324 .

A model of a syntactic pattern recognition-based agent is introduced in the next chapter. An architecture of the multi-agent system and scenarios of its functioning are discussed in chapter 4. The final chapter contains concluding remarks.

\section{Syntactic Pattern Recognition-Based Agents}

We introduce a model of a syntactic pattern recognition-based agent in this chapter. In section 2.1 we present basic phases of a syntactic pattern recognition approach to an analysis of hand postures and a formal model based on ETPL $(\mathrm{k})$ graph grammars 2 4,7,10]. Then, in the same section, we discuss a basic problem concerning a construction of such a grammar for the Polish Sign Language. Introducing a multi-agent architecture of a system allows us just to solve the problem. An internal architecture of a syntactic pattern recognition-based agent that is defined according to a formal model introduced is presented in section 2.2.

\subsection{A Formal Model for a Syntactic Pattern Recognition-Based Agent}

An analysis of hand postures of the Polish Sign Language consists of three main phases. During the first phase of image preprocessing a hand region is identified and its contour is approximated with a polygon as it is shown in Figures: 1(a) and 1(b). The region centroid and the polygon vertices constitute the characteristic points of the image - see Fig. 1(b). The image characteristic points are then used, at the second phase of a graph description generation, for spanning a graph structure, which is depicted in Fig. 1(c), and finally for defining the socalled IE-graph on the basis of this structure - see Fig. 1(d). Let us introduce a formal definition of an IE-graph [4].

Definition 1. An indexed edge-unambiguous graph, $I E$ graph, over $\Sigma$ and $\Gamma$ is a quintuple

$$
g=(V, E, \Sigma, \Gamma, \phi)
$$




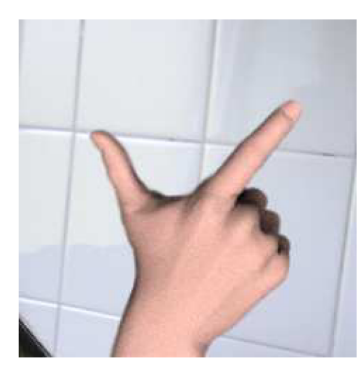

(a)

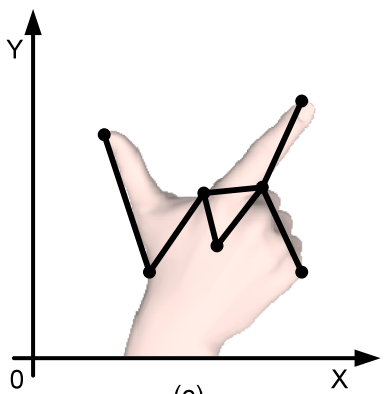

(c)

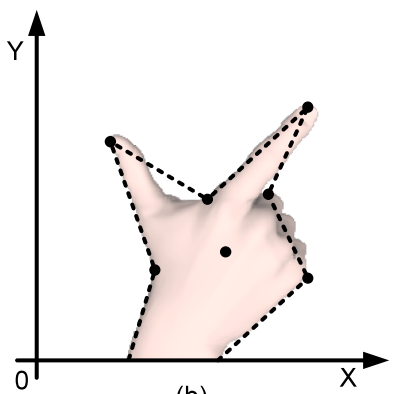

(b)

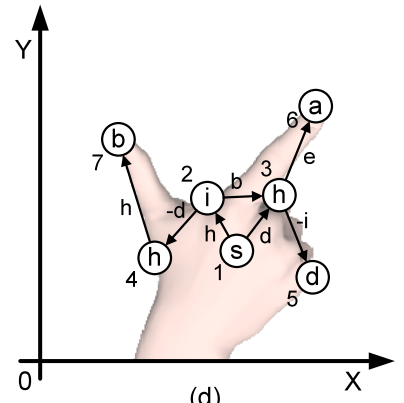

(d)

Fig. 1. Main phases of a syntactic pattern recognition of hand postures of the Polish Sign Language

where $V$ is a finite, non-empty set of nodes that indices have been ascribed to in an unambiguous way,

$\Sigma$ is a finite, non-empty set of node labels,

$\Gamma$ is a finite, non-empty set of edge labels,

$E$ is a set of edges of the form $(v, \lambda, w)$, where $v, w \in V, \lambda \in \Gamma$, such that index of $v$ is less than index of $w$,

$\phi: V \longrightarrow \Sigma$ is a node-labelling function,

and $g$ contains a BFS spanning tree, which nodes are indexed in the same way as nodes of $g$ and its edges are directed in the same way as edges of $g$.

IE-graphs belong to a parsable class of $\operatorname{ETPL}(\mathrm{k})$ graph grammars 247 . The ETPL $(\mathrm{k})$ parsing algorithm is of a very good computational complexity, namely $O\left(n^{2}\right)$ [4. The parsing algorithm uses an ETPL $(\mathrm{k})$ graph grammar (constructed for a given graph language consisting of IE-graphs representing hand postures) as its control table. Of course, constructing such a grammar "by hand" is very difficult in case a language consists of many graphs. Therefore, an inference algorithm generating, automatically, such a grammar on a basis of a set of IEgraphs has been defined and implemented as the INFERGRAPH system [10]. Such a method has occurred to be very efficient.

In case of a recognition of hand postures performed carefully by signers that use a standard (model) Polish Sign Language, a very good recognition rate has been obtained - ca. $95 \%$. However, in case of signers representing a variety of 


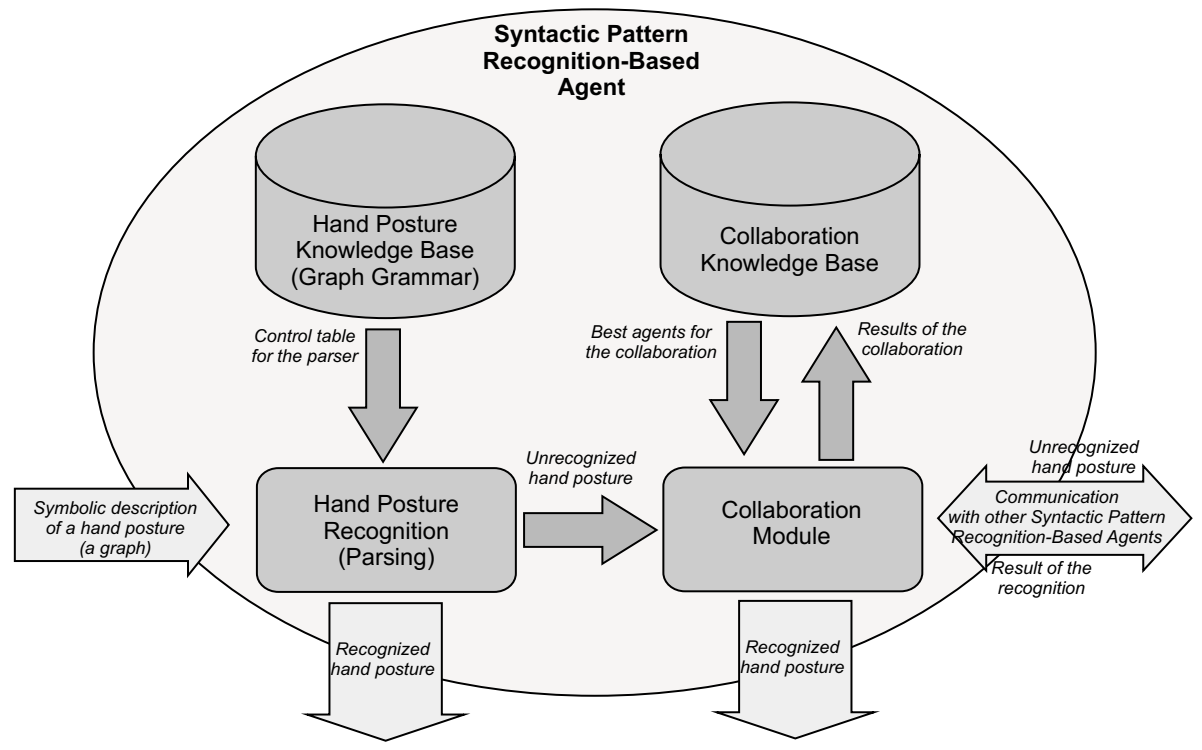

Fig. 2. A general scheme of a syntactic pattern recognition-based agent

(incorrect, negligent) styles (manners), a grammar should contain a lot of productions that differ each from other slightly. This phenomenon makes a definition of a grammar within a parsable class ETPL $(\mathrm{k})$ dubious. In case of an automatic generation of a grammar with the inference system, it can cause a need of introducing a large number of productions that decreases a time efficiency of the method. In extreme cases, it can cause a generation of a grammar within a parsable class ETPL $(\mathrm{k})$ even impossible (if we would like to comprise all the distorted performances of the sign language).

Dividing the whole grammar into sub-grammars corresponding to various styles and manners of a sign language performance allows us to solve a problem discussed above. Then, each such a "specific" sub-grammar is allocated to a syntactic pattern recognition-based agent that is responsible for recognizing a set of hand postures performed in one predefined manner. An architecture of such an agent is discussed in the next section.

\subsection{An Architecture of a Syntactic Pattern Recognition-Based Agent}

There are following main elements of a syntactic pattern recognition-based agent (see Fig. 2):

- a hand posture knowledge base in the form of the ETPL(k) graph grammar describing a manner (variant) of performing hand postures,

- a parser analyses IE-graph representations of hand postures on a basis of the ETPL $(\mathrm{k})$ graph grammar (a hand posture knowledge base), 
- a collaboration module is responsible for: collaborating with other syntactic pattern recognition-based agents (establishing communication, exchanging data, etc.) and accumulating knowledge concerning results of such a collaboration,

- a collaboration knowledge base stores knowledge concerning results of a collaboration with other syntactic pattern recognition-based agents (constantly being updated). Let us notice that the collaboration scenarios are described in details in chapter 3 .

An input for an agent is of the form of an IE-graph being a symbolic representation of a hand posture. A recognition of a hand posture is made with parsing of a received IE-graph (on the basis of an ETPL $(\mathrm{k})$ graph grammar associated with the agent). The result is outputted to the control agent (see: chapter 3).

When an input IE-graph cannot be recognized by the parser, then the agent starts a collaboration with other syntactic pattern recognition-based agents (via collaboration module). The agent sequentially passes the graph to other agents asking for its recognition until it gets a positive answer, i.e. a result of the recognition.

Syntactic pattern recognition-based agents are provided with a self-learning mechanism. Learning is made by a continuous accumulation of knowledge concerning results of the collaboration with other syntactic pattern recognitionbased agents.

Each agent has its own collaboration knowledge base. It is built in the form of the following table.

Table 1. Collaboration knowledge base of an agent

\begin{tabular}{|c|c|c|c|c|}
\hline $\begin{array}{c}\text { Agent } \\
\text { id. }\end{array}$ & $\begin{array}{c}\text { Technical } \\
\text { (communication) } \\
\text { information }\end{array}$ & $\begin{array}{c}\text { Number } \\
\text { of queries }\end{array}$ & $\begin{array}{c}\text { Number } \\
\text { of positive } \\
\text { answers }\end{array}$ & $\begin{array}{c}\text { Percentage } \\
\text { of positive } \\
\text { answers }\end{array}$ \\
\hline A_01 & active, port: $x x x$ & 2 & 1 & $50 \%$ \\
A_02 & inactive, port: $y y y$ & 3 & 0 & $0 \%$ \\
A_03 & active, port: $z z z$ & 10 & 8 & $80 \%$ \\
$\ldots$ & $\ldots$ & $\ldots$ & $\ldots$ & $\ldots$ \\
\hline
\end{tabular}

Information about collaboration with a particular agent is stored in the line of the table. The first column of the table contains technical information needed to communicate with a given agent. The second one contains the number of all queries send to a given agent. The third column contains the number of queries which returned a positive answer (a hand posture has been recognized). The last column contains the percentage of positive answers in case of a given agent.

The table is updated each time the agent asks for the help of another syntactic pattern recognition-based agent. The knowledge included in the table is a basis for a collaboration strategy of the particular agent. A collaboration strategy is described in the next chapter. 
At the end of this section, we would like to underline the autonomy and prosocial behavior of the syntactic pattern-recognition based agents. Therefore, let us discuss how the BDI standards (Belief-Desire-Intention) are embedded in the agents.

- Belief. The belief of an agent is represented by the collaboration knowledge base. Let us notice that the base can be changed. It reflects the fact that what an agent believes may not necessarily be true (it may change in the future).

- Desires. The only desire (goal) of an agent is to recognize a hand posture (being the input data). An agent tries to accomplish the goal by itself or in collaboration with other agents.

- Intentions. The intention of an agent is to recognize a hand posture as quick as possible. If its own parsing algorithm fails, then it decides which other agent is "the best" to cooperate with. The decision is based on the information stored in the collaboration knowledge base (see: section [3.1).

\section{Architecture of the Multi-agent System and Scenarios of its Functioning}

An architecture of the multi-agent system recognizing hand postures is presented in Fig. 3 ,

Let us assume that there are $n$ syntactic pattern recognition-based agents, where each agent is responsible for recognizing hand postures performed in a specific manner. The multi-agent system consists of: several active syntactic pattern

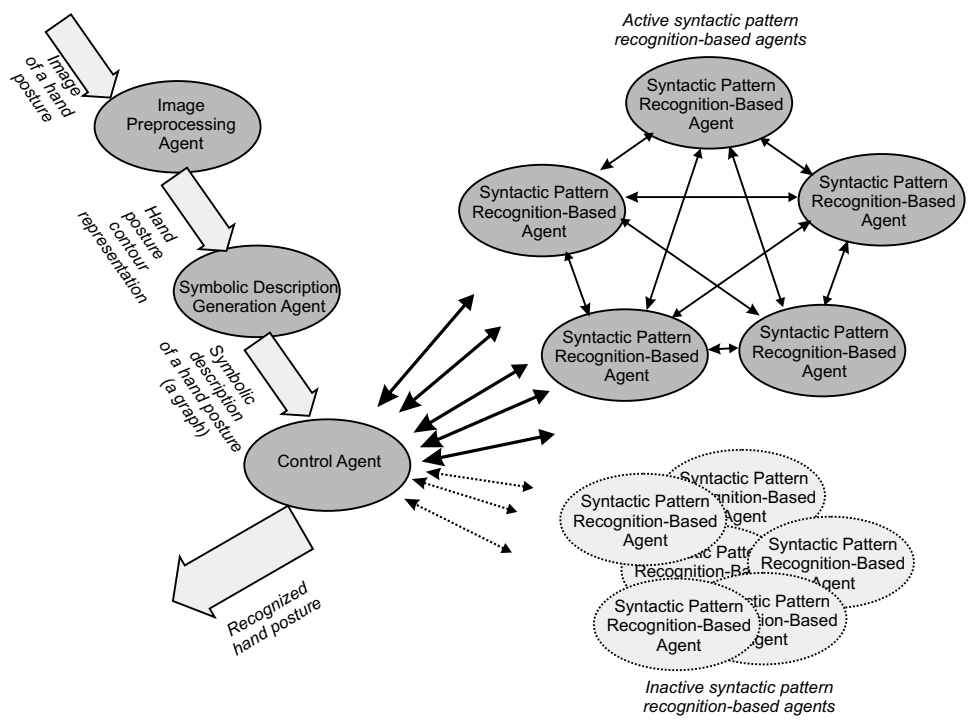

Fig. 3. Architecture of the multi-agent system 
recognition-based agents, the remaining inactive syntactic pattern recognitionbased agents, the control agent, the image preprocessing agent and the symbolic description generation agent (the last two agents are responsible for preliminary phases of image processing that have been discussed in section 2.1).

Active agents are running at processors available for the system. All active agents can communicate with each other. Inactive agents are not allocated to the processors. They wait to be activated by the control agent.

The control agent is responsible for the following tasks:

- a communication with the "outside world" (i.e. collecting hand postures to be recognized, and outputting the results of a recognition),

- an allocation of syntactic pattern recognition-based agents to the processors,

- a communication with syntactic pattern recognition-based agents (a distribution of hand posture IE-graph representations among agents, and gathering results of a recognition),

- an evaluation of a performance of syntactic pattern recognition-based agents and a re-allocation of agents (as a consequence of the evaluation).

In order to perform all tasks mentioned above, the control agent has its own collaboration knowledge base, which is constructed in the same way like the one of a syntactic pattern recognition-based agent (see: Table1). However, a scenario of supplying the base with new information is different from that of syntactic pattern recognition-based agents.

Now, let us present functioning of the system. Initially, the control agent starts as much syntactic pattern recognition-based agents as it is possible in the system. Let $m$ is a number of such agents. Then, the control agent initializes its own collaboration knowledge base and the bases of all active agents (providing information on technical ways of a communication - see: column 2 in Table 10.

In the next step, the control agent begins to receive data from the "outside world". The data are consecutive IE-graph representations of hand postures to be recognized. The agent distributes $m$ hand postures among active syntactic pattern recognition-based agents and waits for their response. The remaining received hand postures are buffered in the FIFO (first-in first-out) structure.

In the next two sections we discuss a further functioning of syntactic pattern recognition-based agents as well as a way of evaluating a performance of these agents and re-allocating these agents made by the control agent.

\subsection{Functioning of Syntactic Pattern Recognition-Based Agents}

There are two following scenarios of functioning of a syntactic pattern recognition-based agent.

Scenario 1. A request to recognize a hand posture is made by the control agent. Firstly, an agent tries to recognize a hand posture itself. An analysis of the hand posture is made by a parser of the agent. If a result of the analysis is positive (the hand posture is recognized), the agent returns information to the control agent which passes it to the "outside world". If the result is negative (the 
hand posture cannot be recognized), the agent starts a collaboration with other syntactic pattern recognition-based agents.

A strategy of a collaboration is straightforward. The agent sorts its collaboration knowledge base accordingly to a percentage of positive answers given by other agents (see: column 4 in Table 1). A request to recognize a hand posture is firstly sent to the "best rated" active agent. If an answer is negative, the request is directed to the next active agent in ranking, and so on. A procedure is repeated until the agent gets a positive answer or all active agents have returned negative answers. During this process the agent collects all information about the collaboration in its knowledge base. Finally, information concerning the responses of the collaborating agents is passed to the control agent.

If a positive answer is finally achieved, it ends the scenario. Otherwise, the agent asks the control agent to activate the remaining agents (which are now in an inactive state) and a recognition process starts again.

Scenario 2. A request to recognize a hand posture is made by another syntactic pattern recognition-based agent.

The agent tries to recognize a hand posture itself, and returns an answer (positive or negative) to an agent that has made the request. In this scenario the agent does not start any collaboration with other agents.

\subsection{Functioning of the Control Agent}

There are two following scenarios of functioning of the control agent.

Scenario 1. A re-allocation as a result of an evaluation of syntactic pattern recognition-based agents.

This is a typical scenario. After receiving an answer from a syntactic pattern recognition-based agent, the control agent updates its collaboration knowledge base, and checks percentages of positive answers for each active agent (in this way the performance of each active agent is evaluated). If there is an active agent $A$ which rank is below a rank of the best inactive agent $N$, then $A$ is replaced with $N$. It means that now $A$ agent becomes inactive, and $N$ agent becomes active and it starts running at a given processor.

Scenario 2. A re-allocation as a result of the lack of a recognition.

The second scenario takes place, if a hand posture cannot be recognized by any active agent in the system. In such an unusual situation, the control agent replaces all active agents with the "best" inactive agents.

\section{Concluding Remarks}

A multi-agent system for an identification of hand postures of the Polish Sign Language has been implemented on the basis of a syntactic pattern recognition approach discussed in the paper. Experimental testing (more than 700 tests) has 
revealed its good characteristics. The average recognition of a hand posture takes $0.13 \mathrm{~s}(\mathrm{C}++$, Intel Core Duo, $1.83 \mathrm{GHz}, 1 \mathrm{~GB} R A M)$, and a recognition rate is of $95 \%$. Including examples of a hand posture performance negligence resulting from specific (incorrect) styles of signing to a sample set makes a graph grammar generating this set very complex and difficult to be maintained.

The main advantage of the use of a multi-agent system approach presented in the paper consists in a possibility of dividing the graph grammar generating IE-graph representations of hand postures into several sub-grammars. It, in turns, allows us to handle a problem of a complexity of a control table of the ETPL(k) parser [4. Such a distributed control table is, obviously, much easier to be maintained with the grammatical inference method [10].

The multi-agent approach not only influences the possibility of constructing a suitable graph grammar (the quality aspect of the system), but also the efficiency of the system. Experiments performed on both single-agent system and 4 -agent system show ca. $28 \%$ increase in time efficiency in favor of the multiagent approach.

There are self-learning mechanisms embedded in a system defined in such a way. Firstly, the control agent learns, which syntactic pattern recognition-based agents provide the best results in case of a particular signer performing hand postures (such agents should use our computing resources firstly, in case of a given signer). Secondly, each syntactic pattern recognition-based agent learns which agents are the best in the collaboration. (It means that the agents can learn the best strategies of a recognition of hand postures). As we have already noticed, styles of showing postures can be combined. It means that an agent that is the best one in a given collaboration is not necessarily the best agent in the global ranking.

A syntactic pattern recognition method presented in the paper is the deterministic one. In case of hand posture images that are distorted (fuzzy), a probabilistic or fuzzy graph grammars would give better recognition rates. It would require to represent also a collaboration knowledge used by the system in a probabilistics (or fuzzy) way. The results of a research into such an extension of the method will be a subject of further publications.

\section{References}

1. Bauer, B., Kraiss, K.F.: Towards an automatic sign language recognition system using subunits. In: Wachsmuth, I., Sowa, T. (eds.) GW 2001. LNCS, vol. 2298, pp. 64-75. Springer, Heidelberg (2002)

2. Flasiński, M.: Parsing of edNLC-graph grammars for scene analysis. Pattern Recognition 21, 623-629 (1988)

3. Flasiński, M., Kotulski, L.: On the use of graph grammars for the control of a distributed software allocation. The Computer Journal 35, A165-A175 (1992)

4. Flasiński, M.: On the parsing of deterministic graph languages for syntactic pattern recognition. Pattern Recognition 26, 1-16 (1993)

5. Flasiński, M.: Use of graph grammars for the description of mechanical parts. Computer Aided Design 27, 403-433 (1995) 
6. Flasiński, M., Schaefer, R., Toporkiewicz, W.: Optimal stochastic scaling of CAE parallel computations. In: Polkowski, L., Skowron, A. (eds.) RSCTC 1998. LNCS, vol. 1424, pp. 557-564. Springer, Heidelberg (1998)

7. Flasiński, M.: Power properties of NLC graph grammars with a polynomial membership problem. Theoretical Computer Science 201, 189-231 (1998)

8. Flasiński, M., Jurek, J.: Dynamically programmed automata for quasi context sensitive languages as a tool for inference support in pattern recognition-based realtime control expert systems. Pattern Recognition 32, 671-690 (1999)

9. Flasiński, M.: Automata-based multi-agent model as a tool for constructing real-time intelligent control systems. In: Dunin-Keplicz, B., Nawarecki, E. (eds.) CEEMAS 2001. LNCS, vol. 2296, pp. 103-110. Springer, Heidelberg (2002)

10. Flasiński, M.: Inference of parsable graph grammars for syntactic pattern recognition. Fundamenta Informaticae 80, 379-413 (2007)

11. Hakeem, A., Shah, M.: Learning, detection and representation of multi-agent events in videos. Artificial Intelligence 171, 586-605 (2007)

12. Holden, E.J., Owens, R.: Visual sign language recognition. In: Klette, R., Huang, T.S., Gimel'farb, G. (eds.) Dagstuhl Seminar 2000. LNCS, vol. 2032, pp. 270-287. Springer, Heidelberg (2001)

13. Hongeng, S., Nevatia, R.: Multi-agent event recognition. In: Proceedings of the Eight International Conference on Computer Vision, Vancouver, Canada, July 0714, vol. 2, pp. 84-91 (2001)

14. Huang, T.S., Pavlovic, V.: Hand gesture modeling, analysis and synthesis. In: Int. Workshop on Automatic Face and Gesture Recognition, Zurich, Switzerland (1995)

15. Julian, V., Carrascosa, C., Rebollo, M., Soler, J., Botti, V.: SIMBA: An approach for Real-Time Multi-agent Systems. In: Escrig, M.T., Toledo, F.J., Golobardes, E. (eds.) CCIA 2002. LNCS (LNAI), vol. 2504, pp. 282-293. Springer, Heidelberg (2002)

16. Jurek, J.: Syntactic pattern recognition-based agents for real-time expert systems. In: Dunin-Keplicz, B., Nawarecki, E. (eds.) CEEMAS 2001. LNCS, vol. 2296, pp. 161-168. Springer, Heidelberg (2002)

17. Jurek, J.: Grammatical inference as a tool for constructing self-learning syntactic pattern recognition-based agents. In: Bubak, M., van Albada, G.D., Dongarra, J., Sloot, P.M.A. (eds.) ICCS 2008, Part III. LNCS, vol. 5103, pp. 712-721. Springer, Heidelberg (2008)

18. Krüger, M., von der Malsburg, C., Würtz, R.P.: Self-organized evaluation of dynamic hand gestures for sign language recognition. In: Würtz, R.P. (ed.) Organic Computing. Springer, Heidelberg (2008)

19. Negnevitsky, M.: Artificial Intelligence. A Guide to Intelligent Systems. AddisonWesley, Reading (2002)

20. Niederberger, C., Gross, M.: Hierarchical and heterogenous reactive agents for realtime applications. Computer Graphics Forum 22, 323-331 (2003)

21. Rodin, V., Benzinou, A., Guillaud, A., Ballet, P., Harrouet, F., Tisseau, J., Le Bihan, J.: An immune oriented multi-agent system for biological image processing. Pattern Recognition 37, 631-645 (2004)

22. Soto, I., Garijo, M., Iglesias, C.A., Ramos, M.: An agent architecture to fulfill real-time requirements. In: Proceedings of the Fourth International Conference on Autonomous Agents, Barcelona, Spain, June 03-07, pp. 475-482 (2000)

23. Würtz, R.P. (ed.): Organic Computing. Springer, Heidelberg (2007)

24. Yang, X., Xing, Y., Nguyen, A.: A Web-Based Face Recognition System Using Mobile Agent Technology. In: The 8th Australian World Wide Web Conference (2002) 